\title{
Observations of dark-cored filaments in sunspot penumbrae
}

\author{
K. Langhans, G. B. Scharmer, D. Kiselman, and M. G. Löfdahl
}

The Institute for Solar Physics of the Royal Swedish Academy of Sciences, AlbaNova University Center, 10691 Stockholm, Sweden e-mail: kai@langhanse.at; [scharmer; dan;mats]@astro.su.se

Received 15 March 2006 / Accepted 16 December 2006

\section{ABSTRACT}

\begin{abstract}
Context. The recent discovery of dark-cored penumbral filaments suggests that we are resolving the building blocks of sunspot penumbrae. Their properties are largely unknown but provide important clues to understanding penumbral fine structure.

Aims. Our observations provide new constraints for the different scenarios put forward to explain the structure of sunspot penumbrae. Methods. We present an analysis of dark-cored penumbral filaments, based on intensity filtergrams $(G$-band, continuum and $\mathrm{Ca}$ II $\mathrm{H}$ line wing), magnetograms and Dopplergrams, obtained at heliocentric distances between $15^{\circ}$ and $55^{\circ}$.

Results. In general, the visibility of dark cores degrades with increasing heliocentric distance. Based on Ca II $\mathrm{H}$ wing images we conclude that this is due to a geometrical 3D-effect and not due to a simple formation height effect. Only in the center-side penumbra are dark-cored filaments visible at all observed heliocentric distances. We observe that dark-cored filaments frequently split in the umbra, forming a Y-shape that disappears after a few minutes, leaving a shortened filamentary structure and a bright dot in the umbra. The dark-cored filaments have life times $\geq 90 \mathrm{~min}$. The dark cores are related to a much weaker and a more horizontal magnetic field than their lateral brightenings. Where the dark-cored filaments appear in the umbra, the magnetic field is inclined by $40^{\circ}$ with respect to the solar surface normal for both the dark core and the bright edges. With increasing distance from the umbra, the magnetic field inclination in the dark cores increases rapidly within a few thousand $\mathrm{km}$. Both the magnetic field strength and inclination in the lateral brightenings show very small variations with spot-center radial distance. The velocity field possesses a strong horizontal component within the dark cores. The absolute line-of-sight (LOS) velocity is larger within the dark cores than in their lateral brightenings. The Evershed flow apparently is present primarily in the dark cores.
\end{abstract}

Key words. Sun: magnetic fields - Sun: photosphere - Sun: sunspots

\section{Introduction}

Much of our current level of understanding of sunspot penumbrae is based on physical (e.g., Meyer \& Schmidt 1968; Thomas 1988; Wentzel 1992; Jahn \& Schmidt 1994; Schlichenmaier et al. 1998a,b) or empirical (e.g., Solanki \& Montavon 1993; Martínez Pillet 2000; Müller et al. 2002; Bellot Rubio et al. 2004; Borrero et al. 2004, 2005, 2006a,b) models to explain penumbral fine structure and flows in terms of flux tubes embedded in a magnetic atmosphere. Such empirical models have in particular been able to explain the net circular polarization (NCP), arising from combined strong gradients in the line-ofsight velocity and magnetic field (Solanki \& Montavon 1993; Martínez Pillet 2000). The success of inversions based on these two-component models in explaining observed Stokes spectra (e.g., Bellot Rubio et al. 2004; Borrero et al. 2004, 2005, 2006a,b) may appear to justify and support the assumed flux tube models; however such data obtained at low spatial resolution do not allow unique interpretations (e.g., Borrero et al. 2004). As pointed out by Spruit \& Scharmer (2006), the two-component flux tube models implemented in the inversion techniques of (Borrero et al. 2004, 2005, 2006a,b) are highly idealized and ignore the strong perturbation of the surrounding magnetic field on the flux tube. Such flux tubes, having circular cross section and a uniform magnetic field inside the flux tube, cannot survive and will be flattened in tens of seconds by the anisotropic forces of the surrounding magnetic field ${ }^{1}$.

1 These problems are high-lighted in the thesis of Borrero (2004, Sect. 6.4). For the parameters chosen, the density at the top of the flux-
A generic problem of all models explaining Evershed flows in terms of steady flows is that such flows must closely follow the $\tau=1$ surface of the penumbra. Furthermore, these flows must be aligned with the magnetic field. Schlichenmaier \& Schmidt (2000), measuring Doppler shifts of two lines formed deep in the photosphere, found that the inclination angles of the measured flows would correspond to flux tubes ascending approximately $300 \mathrm{~km}$ above the formation height of the spectral lines observed. They proposed that this can be explained by averaging over several flux tubes, each bending over and becoming almost horizontal within a small distance from its foot point, but with the foot points of individual flux tubes located at different radii. Bellot Rubio et al. (2003, 2004), applying twocomponent inversions to NIR iron lines formed deep in the photosphere, found strong evidence for field-aligned flows in the flux tube component. However, the inclination of the the flow and magnetic field in the inner penumbra, approximately $70^{\circ}$, shows the same inconsistency with horizontal flux tubes discussed by Schlichenmaier \& Schmidt (2000) and is explained by the same averaging effect. Borrero et al. (2005) also analyzed spectra from NIR lines formed deep in the photosphere and found for a sunspot observed close to disk center that the inclination of the magnetic field is close to $70^{\circ}$ and increasing gradually to about $90^{\circ}$ at the outer boundary of the penumbra. The very small spread of the measured inclinations in the inner penumbra suggests that more than a few flux tubes must be present within the $1^{\prime \prime}$ resolution element to be consistent with the

tube must be higher than that of the photosphere and negative at the bottom of the flux tube in order to prevent collapse of the flux tube. 
averaging effect proposed by Schlichenmaier \& Schmidt (2000). Recently, Rimmele \& Marino (2006) analyzed a 120 min long Dopplergram time sequence obtained in the non-magnetic neutral iron line at $557.6 \mathrm{~nm}$. The very high spatial resolution of this data allowed the identification of individual flow channels and their foot-points, co-aligned with bright grains (peripheral umbral dots). Rimmele \& Marino concluded that the upflows turn into horizontal flows within fractions of an arcsecond but present no analysis of the azimuthal variation of the measured line-of-sight velocities, needed to justify this conclusion.

The discovery of dark cores in penumbral filaments (Scharmer et al. 2002; Rouppe van der Voort et al. 2004; Sütterlin et al. 2004; Langhans et al. 2005; Langhans 2006; Rimmele \& Marino 2006) suggests that we are starting to resolve the fundamental scales of the penumbra and that these cores provide important clues to understanding its internal structure (Scharmer et al. 2002), while also posing new problems. As shown by Schlichenmaier \& Solanki (2003), the energy flux associated with upflow channels similar to those of Schlichenmaier et al. $(1998 \mathrm{a}, \mathrm{b})$ cannot provide the energy needed to explain the radiative flux of the penumbra for a filament longer than approximately $1200 \mathrm{~km}$, assuming a flux tube diameter of less than $200 \mathrm{~km}$. Schlichenmaier \& Solanki (2003) concluded that such flux tubes must submerge within 1000-2000 km from their foot points in order to give room for new flow channels. Relying on simulations by Schlichenmaier (2002), Schlichenmaier \& Solanki (2003) suggested that the submerged part of the flux tube is heated radiatively by hotter gas below the photosphere, causing it to reappear as a hot upflow channel. However, Rouppe van der Voort et al. (2004) analyzed dark-cored filaments with lengths of over $6000 \mathrm{~km}$ without any indication of fine structure consistent with such aligned (reappearing) upflow channels. Schlichenmaier \& Solanki (2003) also concluded that the long life times of observed filaments excludes the possibility of efficient interchange convection (cf. Jahn \& Schmidt 1994) to heat the penumbra.

Spruit \& Scharmer (2006) pointed out that the embedded flux tubes assumed to explain penumbral fine structure and flows constitute a hindrance to explanations of penumbra heating and proposed a new model in which convection below the visible surface in radially aligned field-free gaps is responsible for the filamentary structure. The large vertical magnetic field gradients are in this model explained as a natural consequence of the fieldfree gaps, the dark cores as signatures of an elevated $\tau=1$ surface above the field-free gap combined with an overall drop of temperature with height. The existence of elevated filaments was also suggested by Schmidt \& Fritz (2004) based on measurements of the center-to-limb and azimuthal variation of the penumbral intensity for a large number of spots.

The identification of penumbra filaments and their dark cores with embedded flux tubes harboring steady Evershed flows requires that the observed flows and magnetic fields are sufficiently horizontal. The dark cores in penumbral filaments have been shown by Rimmele \& Marino (2006) to outline flow channels. In this paper we present filtergrams obtained in the $G$-band, the nearby continuum at $436.4 \mathrm{~nm}$ and in the Ca II $\mathrm{H}$ line wing as well as magnetograms and Dopplergrams of sunspots, observed for a large range of different heliocentric angles $\left(15^{\circ}\right.$ to $\left.55^{\circ}\right)$. These observations obtained at high spatial resolution provide direct information about the magnetic field and the flow field of dark-cored penumbral filaments. Furthermore, we compare the visibility of dark cores observed in the $\mathrm{Ca}$ II $\mathrm{H}$ line wing, formed $150-200 \mathrm{~km}$ above the photosphere, with continuum and $G$-band images recorded outside disk center. This allows us to establish
Table 1. Data pairs: C4364 - broad band continuum at $436.39 \mathrm{~nm}$. C6302 - narrow band continuum images, obtained with the SOUP at $-35 \mathrm{pm}$ from the line core of Fe I $630.2 \mathrm{~nm}$ line. GB $-G$-band filtergram. $\mathrm{CaH}$ - filtergram in the Ca II H line wing at $396.4 \mathrm{~nm}$. CS - Scan through the blue line wing of Ca II H. M - magnetogram in the blue wing of Fe I $630.2 \mathrm{~nm}$ D6302 - 2-point Dopplergram obtained in the wings of Fe I $630.2 \mathrm{~nm}$. D5576 - 2-point Dopplergram obtained in the wings of Fe I $557.6 \mathrm{~nm}$. D 5576 - 4-point Dopplergram.

\begin{tabular}{|c|c|c|}
\hline \multicolumn{3}{|c|}{ large less symmetric sunspot, leading spot of NOAA 10397: } \\
\hline July 7,2003 & $\mu=0.57$ & C6302+M+D6302 \\
\hline \multicolumn{3}{|c|}{ small symmetric sunspot, leading spot of NOAA 10425 : } \\
\hline August 8, 2003 & $\mu=0.96$ & $\mathrm{C} 4364+\mathrm{GB}+\mathrm{CaH}+\mathrm{M}$ \\
\hline August 9, 2003 & $\mu=0.88$ & $\mathrm{C} 4364+\mathrm{GB}+\mathrm{CaH}+\mathrm{M}+\mathrm{D} 6302$ \\
\hline \multicolumn{3}{|c|}{ large symmetric sunspot of NOAA 10644: } \\
\hline July 16,2004 & $\mu=0.95$ & $\mathrm{C} 6302+\mathrm{M}+\mathrm{D} 6302+\mathrm{D} 5576+\mathrm{CS}$ \\
\hline July 20, 2004 & $\mu=0.57$ & $\mathrm{C} 6302+\mathrm{M}+\mathrm{D} 6302+\mathrm{D} 5576$ \\
\hline \multicolumn{3}{|c|}{ large sunspot, leading spot of NOAA 10649: } \\
\hline July 16,2004 & $\mu=0.87$ & $\mathrm{C} 6302+\mathrm{M}+\mathrm{D} 6302+\mathrm{D} 5576+\mathrm{CS}$ \\
\hline \multicolumn{3}{|c|}{ irregular sunspot of NOAA 10653: } \\
\hline July 20,2004 & $\mu=0.75$ & $\mathrm{C} 6302+\mathrm{M}+\mathrm{D} 6302+\mathrm{D} 5576$ \\
\hline \multicolumn{3}{|c|}{ less symmetric sunspot, leading spot of NOAA 10655: } \\
\hline August 5, 2004 & $\mu=0.96$ & $\mathrm{C} 6302+\mathrm{M}+\mathrm{D} 6302+\mathrm{D}^{4} 5576$ \\
\hline August 6, 2004 & $\mu=0.90$ & $\mathrm{C} 6302+\mathrm{M}+\mathrm{D} 6302$ \\
\hline August 8, 2004 & $\mu=0.59$ & $\mathrm{C} 6302+\mathrm{M}+\mathrm{D} 6302+\mathrm{D}^{4} 5576$ \\
\hline August 9, 2004 & $\mu=0.45$ & $\mathrm{C} 6302+\mathrm{M}+\mathrm{D} 6302+\mathrm{D}^{4} 5576$ \\
\hline
\end{tabular}

that the visible $\tau=1$ surface of the mid-inner penumbra, where dark cores are observed, is strongly warped.

\section{Observations}

All observations were acquired with the SST (Scharmer et al. 2003a) at the Observatorio del Roque de los Muchachos (La Palma, Spain) using its adaptive optics system (Scharmer et al. 2003b). For the analysis, we use data sets of several spots observed during July and August 2003 and July and August 2004 as listed in Table 1. The small symmetric spot of NOAA 10425 was also observed on August 9, 2003 with the Dutch Open Telescope and used by Sütterlin et al. (2004) for their analysis of dark-cored filaments.

The filtergrams of circular polarized light in the wings of the Fe I line at $630.2 \mathrm{~nm}$ (5 pm from line center) as well as the filtergrams in the wings of the Fe I line at $557.6 \mathrm{~nm}$ were acquired with the Lockheed Solar Optical Universal Polarimeter (SOUP) tunable birefringent filter (cf. Berger \& Title 2001) preceded by an alternating left/right circular polarization (LCP/RCP) filter as well as a blocking filter. For the observations obtained in 2004, the polarization filters were replaced by two liquid crystals. The SOUP filtergrams were obtained with exposure times of 90 to $180 \mathrm{~ms}$. The plate scale is $0{ }^{\prime} 075 / \mathrm{pixel}$. In parallel with the SOUP filter data sets, we obtained also filtergrams in the blue continuum ( $436.4 \pm 0.57 \mathrm{~nm}, 17$ to $22 \mathrm{~ms}$ integration time), in the $G$-band ( $430.5 \pm 0.54 \mathrm{~nm}, 20$ to $28 \mathrm{~ms})$ and in the blue wing of the Ca II H line (396.1 nm, 40 to $50 \mathrm{~ms}$ ). Additionally, on July 16, 2004 we obtained filtergrams at different wavelength positions in the blue wing of the $\mathrm{Ca}$ II $\mathrm{H}$ line. These scans were obtained by tuning a $0.11 \mathrm{~nm}$-wide Ca II $\mathrm{H}$-line interference filter, mounted on a rotatable stage. The plate scale of these 
filtergrams is 0 . $^{\prime} 041 /$ pixel for the 2003 data and $0.0385 /$ pixel for the 2004 data.

All images were obtained in real-time frame selection mode. The selected frames were post-processed using Multi-Frame Blind Deconvolution (MFBD; Löfdahl 2002). For the $G$-band, the continuum, and the Ca II $\mathrm{H}$ filtergrams, the best 3 to 5 frames within $12 \mathrm{~s}$ were selected. To obtain almost simultaneous magnetic information from the Fe I $630.2 \mathrm{~nm}$ filtergrams, we select 3 to 5 frames within each $5 \mathrm{~s}$ time interval before changing the polarization state. In the same manner, filtergrams in the line wings of the $\mathrm{Fe}$ I line at $557.6 \mathrm{~nm}$ were taken to obtain information about the LOS velocity. For some data sets, filtergrams alternating between the red and the blue wings of Fe I $630.2 \mathrm{~nm}$ were recorded to obtain additional information about the LOS velocity. All data sets included in the analysis are listed in Table 1.

\section{Data reduction and analysis}

The data calibration is described in detail in an earlier paper (Langhans et al. 2005, hereinafter Paper I).

We computed the magnetograms, $M^{\vartheta}$, and Dopplergrams, $V_{6302}^{\vartheta}$ and $V_{5576}^{\vartheta}$, in the following manner:

$M^{\vartheta}=\frac{I_{-5}^{\mathrm{LCP}}-I_{-5}^{\mathrm{RCP}}}{I_{-5}^{\mathrm{LCP}}+I_{-5}^{\mathrm{RCP}}}$,

$V_{6302}^{\vartheta}=f_{\mathrm{c}, 6302} \frac{\left(I_{-5}^{\mathrm{LCP}}+I_{-5}^{\mathrm{RCP}}\right)-\left(I_{+5}^{\mathrm{LCP}}+I_{+5}^{\mathrm{RCP}}\right)}{I_{-5}^{\mathrm{LCP}}+I_{-5}^{\mathrm{RCP}}+I_{+5}^{\mathrm{LCP}}+I_{+5}^{\mathrm{RCP}}}$,

and

$V_{5576}^{\vartheta}=f_{\mathrm{c}, 5576} \frac{I_{-5}-I_{+5}}{I_{-5}+I_{+5}}$.

Four-point Dopplergrams were obtained as described by Shine et al. (1994):

$V_{4}^{\vartheta}=2055 \arctan \left(\frac{I_{+3}-I_{-9}+I_{+9}-I_{-3}}{I_{-9}-I_{-3}-I_{+3}+I_{+9}}\right)$,

where the indices give the distance from the center wavelength of the corresponding line in pm. We refer to $M^{\vartheta}$ as the magnetogram signal, where $\vartheta$ indicates the LOS angle. The observed spots in NOAA 10397, 10649, 10653 and 10655 have opposite polarity to that of NOAA 10425 and 10644. For clarity in images and diagrams, the magnetogram signal of those spots is inverted.

The calibration of the Dopplergrams, i.e., the estimation of the calibration functions $f_{\mathrm{c}}$, is described in Paper I along with a discussion of the influence of velocity fields on the magnetogram signal and the temperature dependence of the magnetogram signal. The sign convention in $f_{\mathrm{c}}$ is selected such that positive velocities represent red-shifts.

In order to establish the zero point of the Dopplergrams, we made calibrations based on the assumption of an umbra at rest and based on the surrounding photosphere with a correction for the convective blue shift applied (based on Bellot Rubio et al. 2004; Balthasar 1985). The zero-point velocities obtained with the two methods differ by up to several hundred $\mathrm{m} \mathrm{s}^{-1}$. The differences may have several explanations: (i) lack of an adequate umbral reference (size of the umbra, low signal-to-noise ratio in the umbra); (ii) influence of the moat flow in the reference region outside the spot; (iii) incorrect determination of the line-center position during the SOUP calibration or wavelength drifts during the observations. The zero point velocities finally adopted are average values obtained from the umbra and quiet sun calibrations and may have errors as large as $\pm 250 \mathrm{~m} \mathrm{~s}^{-1}$.

To create the time series of the data obtained on August 8, 2003 , we aligned all $G$-band images temporally, including destretching to compensate for anisoplanatism. Next, each filtergram in the other wavelengths was aligned to the $G$-band frame nearest in time. The aligned frames were clipped to the common field-of-view of the polarimetric and the other filtergram data $\left(74^{\prime \prime} \times 74^{\prime \prime}\right)$. Finally, the data cubes were time-space filtered to attenuate brightness oscillations from the 5-min p-modes by using a subsonic Fourier filter (Title et al. 1989) The filter removes modulations with horizontal speeds above a threshold of $4 \mathrm{~km} \mathrm{~s}^{-1}$. The fact that the filtergrams in LCP and RCP light are not obtained simultaneously represents a major potential error source (cf. Paper I) and the analysis was restricted to magnetograms and Dopplergrams made from the very best filtergrams.

\section{Results}

As described by, e.g., Sütterlin et al. (2004) and Bellot Rubio et al. (2005), dark cores are best visible and easiest to identify where the penumbral filamentary structure is orientated along the symmetry line from spot center to solar disk center. We therefore restricted the analysis to subfields of the center- and the limb-side penumbra. For these subfields, we trace intensity, magnetogram signal, and Doppler velocity, respectively, across and along selected dark-cored penumbral filaments. The corresponding paths are marked in the accompanying filtergrams.

\subsection{General observations}

Figure 1 shows dark-cored filaments of the small circular sunspot of NOAA 10425 at $\mu=0.96$ in the blue continuum, in the $G$-band, in the wing of the Ca II H-line, along with the corresponding magnetogram. Figure 2 is similar, showing continuum and magnetograms at $\mu=0.88$. Dark-cored filaments look very similar in the continuum intensity $(436.4 \mathrm{~nm})$ and the $G$-band intensity (Figs. 1a and b). The diagrams in Fig. 1c show that the variations of the normalized intensity across dark-cored filaments are of the same magnitude in the $G$-band and in the continuum for both the center- and limb-side penumbra. In general, dark cores are very pronounced and can be identified over a large radial part, especially in the center-side of the penumbra. But in the innermost part of the limb-side penumbra, the contrast of the dark-cored filaments is larger than in the center-side penumbra. The intensity plots in Figs. 1c and 2d, show that the lateral brightenings peak at higher intensities in the limb-side than in the center-side penumbra. Calculating the rms intensity contrast within a half arcsecond-wide subfield centered around the selected paths gives a contrast value of 0.20 for the limb side and 0.12 for the center side. The average continuum intensity is higher in the limb side $(0.74)$ than in the center center side (0.70). At the penumbral-umbral border of the limb-side penumbra, the dark-cored filaments are associated with dot-like brightenings that are related to a strong positive magnetic signal. A close-up view of such brightenings, visible both the continuum filtergram and the magnetogram, is shown in Fig. 2c. This signature is not visible in the center-side penumbra.

Overlaying an intensity filtergram and a magnetogram shows that the dark-cored filament as identified in the filtergram and the coherent radially extending structure observed in the magnetogram indeed represent the same structure. The magnetograms associated with such dark-cored filaments show coherent radial structures, consisting of a low-signal component flanked on both 


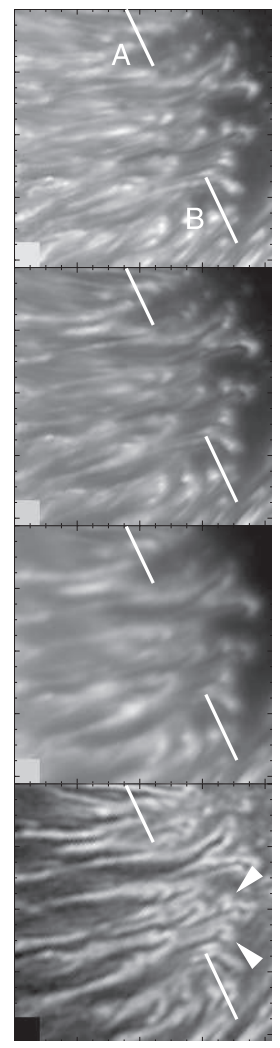

(a) center side, CS

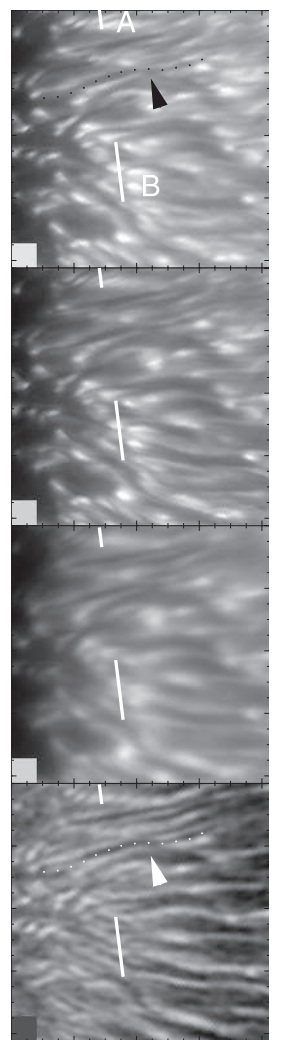

(b) limb side, LS
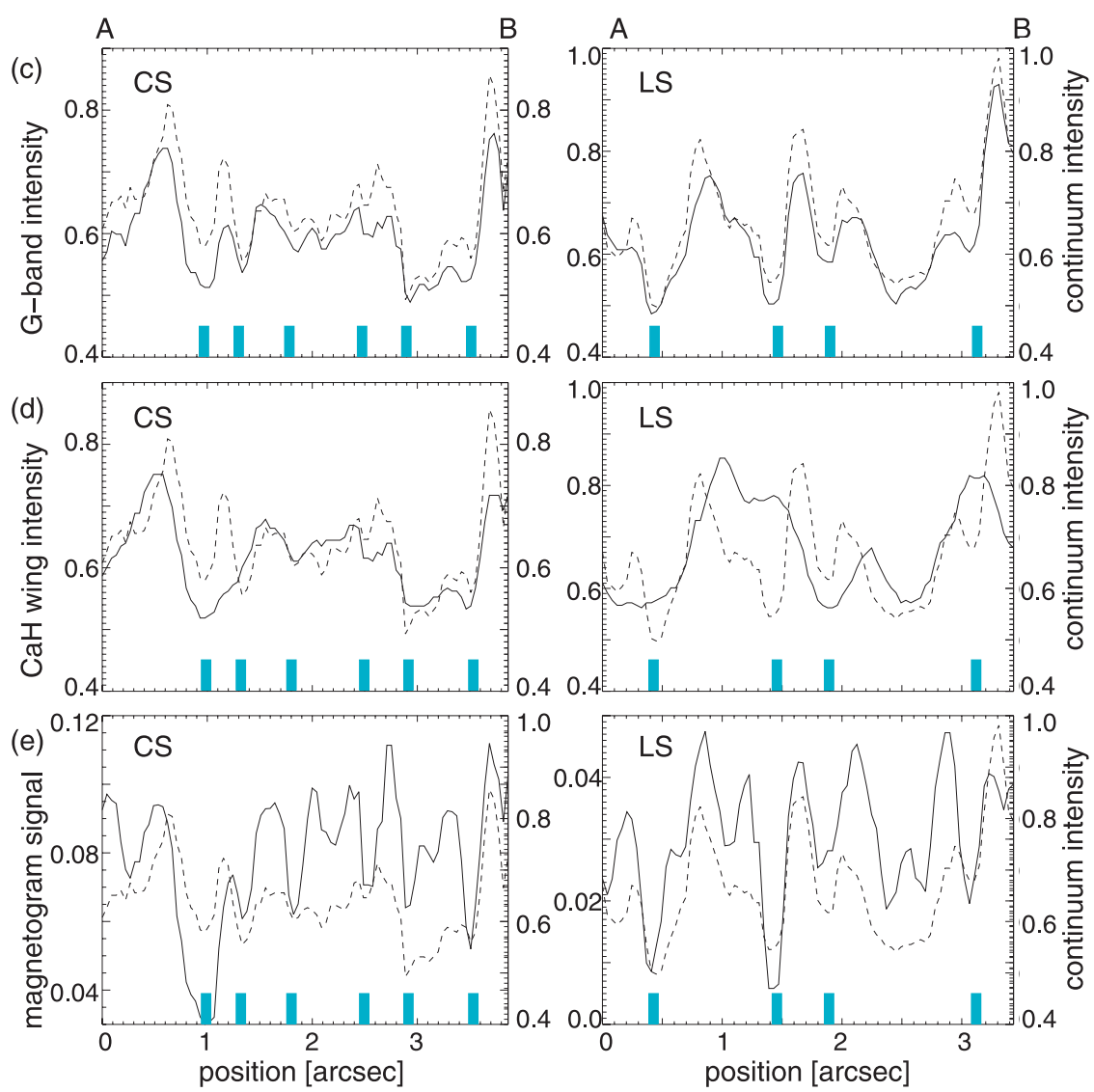

Fig. 1. NOAA 10425 on August 8, 2003, LOS angle $16^{\circ}$. a) Center-side penumbra (top to bottom: continuum, $G$-band, Ca II H wing, magnetogram), b) Limb-side penumbra. c) $G$-band intensity along the cut A-B as indicated in the maps. The left column refers to the center-side, the right column to the limb-side penumbra. d) Intensity in the blue wing of Ca II H line (396.4 nm). e) Magnetogram signal. The dashed curves represent the continuum intensity at $436.4 \mathrm{~nm}$, normalized to quiet sun intensity (scale to the right). The squares in the lower left of the magnetograms mark the grey scale corresponding to a magnetic signal of zero. The grey bars in the diagrams indicate the positions of the identified dark cores.

sides by high signal components. Often these compound magnetic structures are more distinct and can be followed much further out from the inner penumbra than the dark cores seen in filtergrams. In the following, we refer to these magnetic structures as dark-core magnetic structures. In Fig. 1, we indicate a dark core by a black-dotted line in the continuum and a white-dotted line in the magnetogram, respectively. Tracing the filament from the inner penumbra outward, the lateral brightenings appear to disappear at the position indicated by the arrow, but reappear further out. In contrast, the dark-core magnetic structure shows a radially coherent structure across the position marked by the arrow. In general, variations in brightness of the lateral bright components along the dark-cored filaments in the intensity filtergrams are not reflected in the magnetograms. Nor do we find a counterpart in magnetograms and Dopplergrams to the asymmetry of individual dark-cored filaments observed in intensity filtergrams as described by Scharmer et al. (2002). For example, the dark-cored filament in Fig. 1 at 3.' 5 shows a strong magnetic signal on both sides of the dark core but the intensity is much higher on one side than on the other.

The visibility of dark-cored filaments decreases with increasing LOS angle $\vartheta$. In the limb-side penumbrae of the spots observed at about $\mu=0.57$ (NOAA 10397, July 7, 2003 and NOAA 10644, July 20, 2004) we can only identify a few dark cores. In the magnetograms the dark-cored magnetic structures are visible also at other azimuth angles, but are more distinct close to the symmetry line. (cf. Fig. 3 in Paper I for a symmetric sunspot at a heliocentric angle of $28^{\circ}$.)
Irrespective of the azimuth angle, we find that dark cores (where such can be identified) consistently are related to a reduced magnetogram signal compared to their immediate surroundings. Figures $1 \mathrm{a}, \mathrm{b}$ and $2 \mathrm{a}, \mathrm{b}$ show that the dark core magnetic structures are more distinct and easier to trace outwards than the dark cores seen in continuum or $G$-band images.

Figure 3 illustrates the variation of the continuum intensity and magnetogram signal along a typical dark-cored filament at different distances from the umbra (cuts A to G). The displayed filaments split at the umbra-penumbra border forming a Y-shape, that is a common aspect of dark-cored filaments (Scharmer et al. 2002). Additional examples are indicated by arrows in the magnetogram of Fig. 1a. The variation in intensity and magnetogram signal across the "tails" (A) demonstrates that each "tail" that extends into the umbra shows the same variation as two separated individual dark-cored filaments. The dark core can be identified throughout the whole penumbra, both in intensity and magnetogram signal. The intensity drop in the dark core is largest in the inner penumbra $(0.3$ in units of the normalized continuum intensity). The dark core intensity decreases with increasing distance from the umbra. The distance between the two lateral brightenings - defined as the width of dark-cored filaments by Scharmer et al. (2002) and Sütterlin et al. (2004) - increases slightly with increasing distance, from 0.'3 (A) to above $0 !^{\prime} 4(\mathrm{G})$. At a given distance from spot center, the components of a dark-cored filament differ in their magnetogram signals. The bright edges have a stronger magnetogram signal than the dark cores over the entire penumbra. The magnetogram 


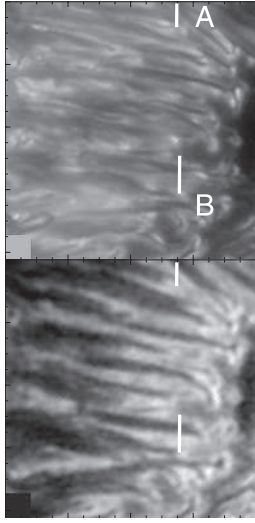

(a) center side, CS

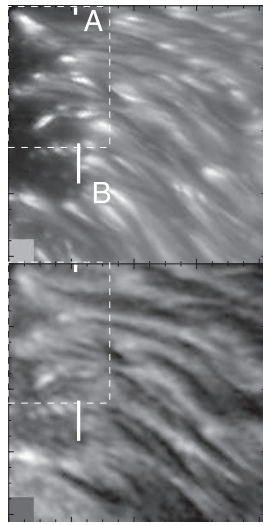

(b) limb side, LS (c)
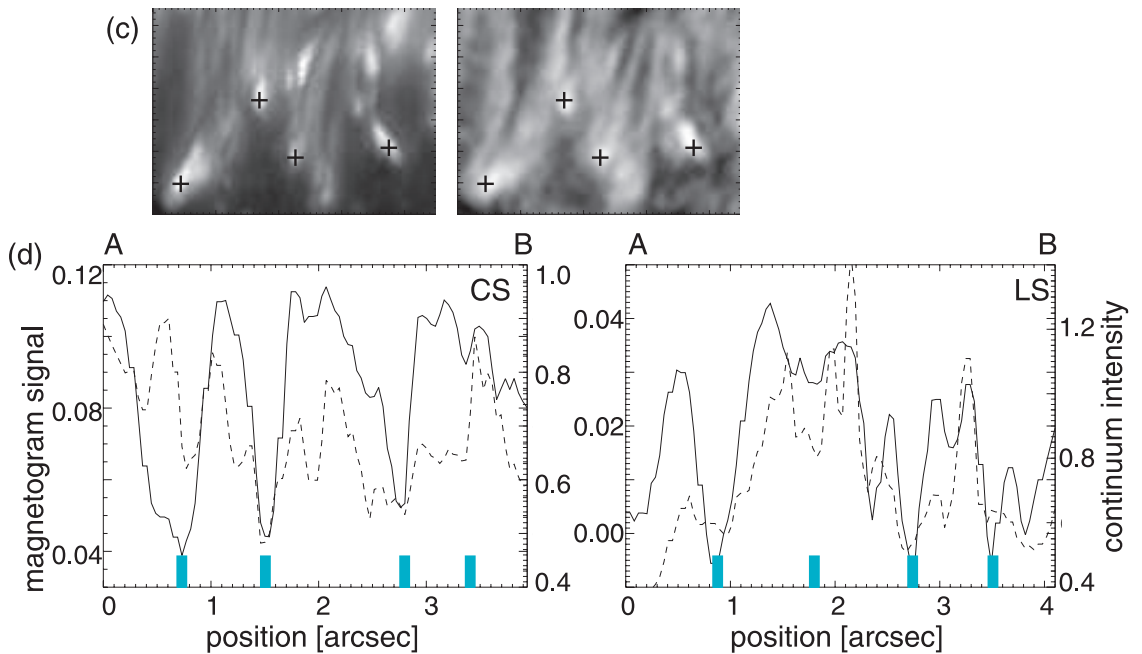

Fig. 2. NOAA 10425 on August 9, 2003, LOS angle $28^{\circ}$. a) Center-side penumbra (top: continuum, bottom: magnetogram), b) Limb-side penumbra. c) Close-up view (rotated by $90^{\circ}$ counter-clockwise) of the subfield indicated by the dashed lines in b). Dot-like brightenings are indicated with + symbols. d) Magnetogram signal along the cut A-B as indicated in the maps. The dashed curves represent the continuum intensity at $436.4 \mathrm{~nm}$, normalized to quiet sun intensity (scale to the right). The squares in the lower left of the magnetograms mark the grey scale corresponding to a magnetic signal of zero. The grey bars in the diagrams indicate the positions of the identified dark cores.
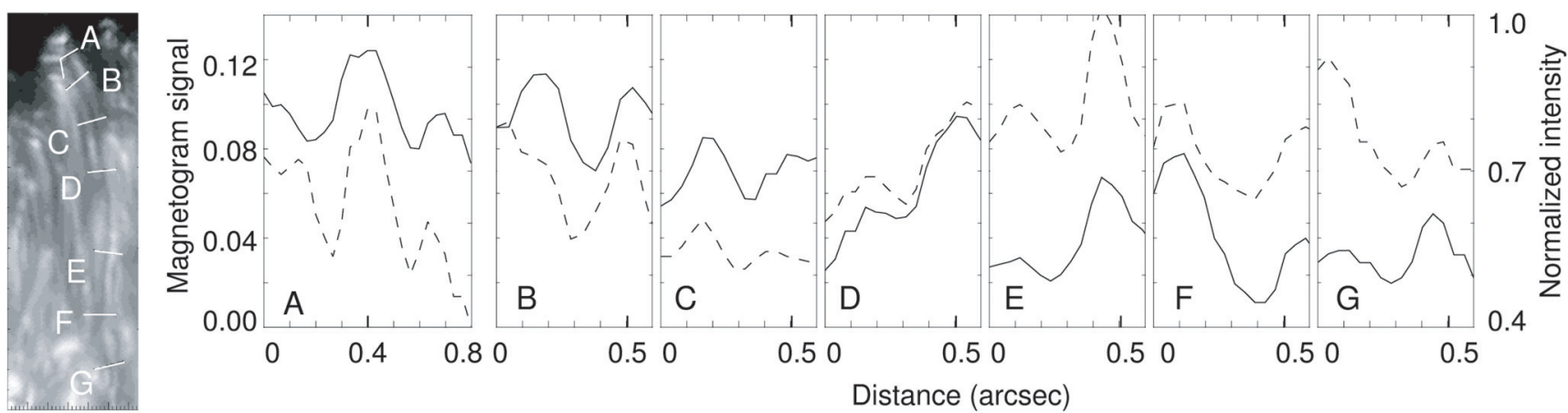

Fig. 3. Variation of continuum intensity and magnetogram signal throughout the center-side penumbra along a dark-cored filament (NOAA 10425 on August $8, \operatorname{LOS}$ angle $16^{\circ}$. The solid curves represent the magnetogram signal (scale to the left), the dashed curve represents the continuum intensity at $436.4 \mathrm{~nm}$, normalized to quiet sun intensity (scale to the right).

signal decreases for both dark cores and lateral edges toward the outer penumbra.

\section{2. $\mathrm{Ca} / \mathrm{H}$ line-wing filtergrams}

The Ca II H wing is formed close to LTE, and scanning through it shows - with some complications - the kinetic temperature as a function of column density (cf. Rouppe van der Voort 2002). The large opacity of this (and the Ca II K) line, combined with an insensitivity to Doppler shifts, provide a unique diagnostic in the visible solar spectrum. In the absence of a quantitative analysis, we inspect the filtergrams (A to D) in Fig. 4 which were obtained at different positions in the wing. Very roughly, they correspond to the following indicative mean formation heights (Rouppe van der Voort, private communication) over the continuum level of the VAL3C model (Vernazza et al. 1981). A: $170 \mathrm{~km}, \mathrm{~B}: 100 \mathrm{~km}, \mathrm{C}: 60 \mathrm{~km}, \mathrm{D}: 10 \mathrm{~km}$. In comparison, the $G$-band images are formed at a height of approximately $50 \mathrm{~km}$ above the quiet sun photosphere (Carlsson et al. 2004), corresponding to the height of the filtergrams shown in Fig. 4C. We note that inverted granulation is clearly seen in A, confirming that this passband shows temperature structure well above the photosphere, and that even B shows a fading granulation pattern, distinctly different from what is seen in continuum and $G$-band images. However, the fine structure seen in the penumbra looks essentially the same in all passbands A-D. The main difference between the penumbra shown in A and D is that dot-like structures in D cannot be identified in A and that close to line center, the fine structure is more diffuse than further out in the wing.

In Fig. 4 is shown the variation of the $\mathrm{Ca}$ II H-line wing intensity along three dark-cored filaments for the different passbands observed. In these plots, the different passbands are indicated by the thickness of the curves - with the thickness increasing with distance from the line center. The intensity is normalized with respect to the photosphere outside the spot. The plots that display the variation of the $\mathrm{Ca}$ II $\mathrm{H}$-line wing intensity along the cut A-B (Fig. 1d) show that most dark cores that are visible in the continuum can be identified also in filtergrams obtained in the $\mathrm{Ca}$ II $\mathrm{H}$-line wing. Some of the intensity minima in $\mathrm{Ca}$ II $\mathrm{H}$ seem to be shifted in comparison to the position of the related dark cores as observed in the continuum intensity, others appear unresolved. In general, the intensity contrast of dark-cored filaments is somewhat lower in the Ca II H-line wing than in the continuum. This may be due to lower resolution from the longer exposure times.

The Ca II H images map kinetic temperature at continuum optical depths that increase with the distance from line center for the passband observed. This allows us to immediately 

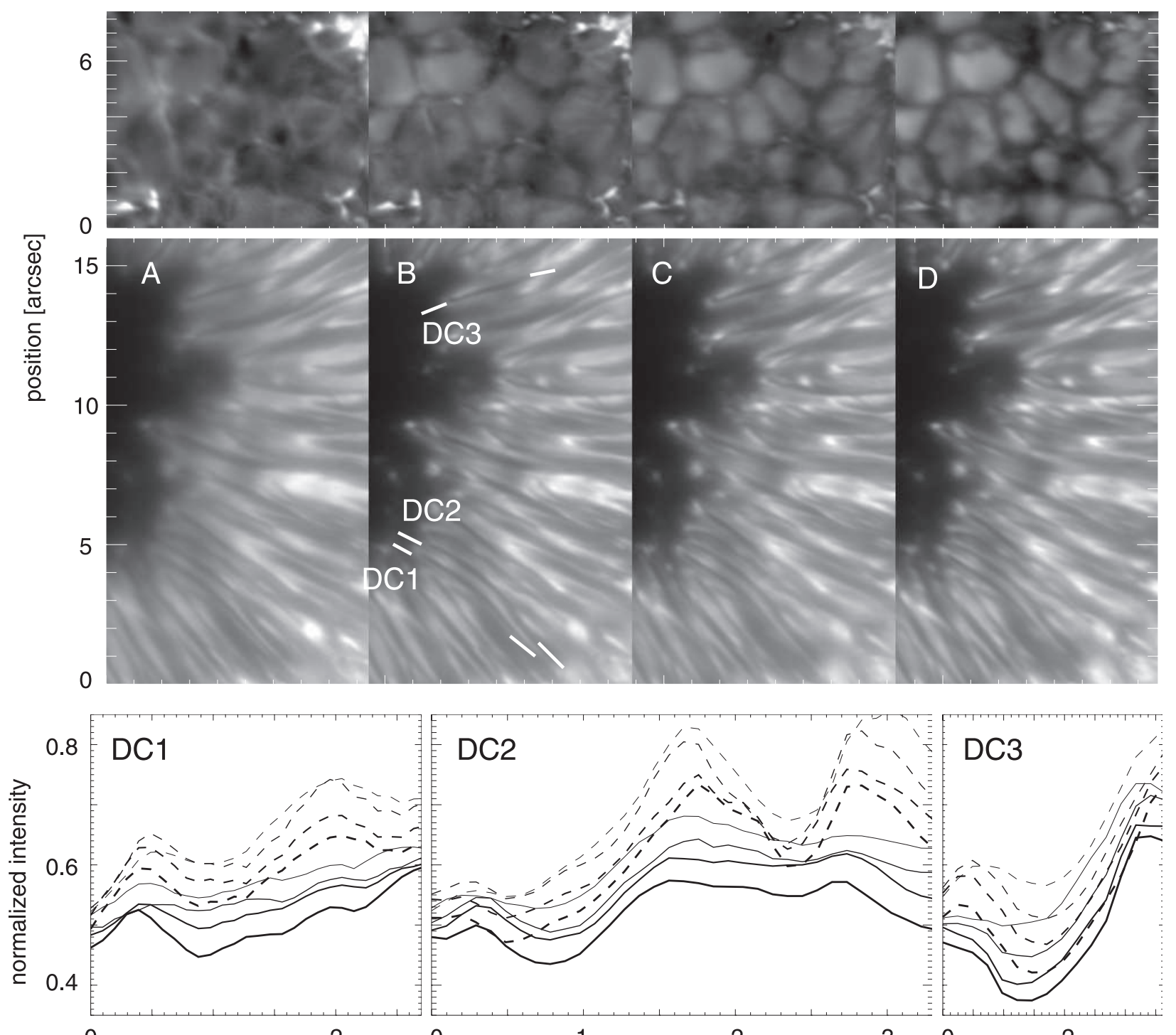

position along dark core [arcsec]

Fig. 4. NOAA 10644 on July 16, 2004, LOS angle $10^{\circ}$. Top: filtergrams obtained at different positions in the blue Ca II $\mathrm{H}$ line wing - increasing distance from line center from left to right (A: $0.2 \mathrm{~nm}, \mathrm{~B}: 0.3 \mathrm{~nm}, \mathrm{C}: 0.4 \mathrm{~nm}, \mathrm{D}: 0.7 \mathrm{~nm}$ ). The upper panel shows granulation close to the spot as reference. Bottom: variation of the normalized Ca II H-line wing intensity along dark cored filaments. Solid curves present the intensity variation along the dark cores, the dashed curves along the lateral brightenings, respectively. The line thickness represents the line wing position where the filtergram is taken. Increasing thickness means increasing distance from line center (corresponding to A to D in the top panel).

conclude that dark cores remain cool relative to their surroundings at heights corresponding to continuum optical depths in the range $0.1-1$. However, radiative transfer diagnostics alone do not allow any conclusions about temperature variations at a fixed height. The measured intensity (temperature) variations at fixed optical depths may be due to horizontal temperature variations at fixed height, but can also be due to purely vertical temperature variations combined with surfaces of constant optical depth that vary strongly in height with position in the spot, i.e., a warped visible surface. Evidence for a warped visible surface comes from continuum images of dark cores observed off disk center. Such images show that dark cores can hardly be seen, except in the disk center direction, already when $\mu=0.57$. The Eddington-Barbier relation for a plane-parallel atmosphere implies that this radiation reaches us from a continuum optical depth of $\tau \approx \mu=0.57$, i.e., much deeper down than the formation height corresponding to panel A in Fig. 4. This confirms what already the azimuthally varying visibility of dark-cored filaments suggests: that the dark-cored filaments are associated with strongly warped surfaces of constant optical depth. This is discussed further in Sect. 5.

\subsection{Dark-cored filaments observed at different heliocentric positions}

Figure 5 shows parts of the center- and limb-side penumbrae of sunspots observed at different heliocentric angles between $15^{\circ}$ and $55^{\circ}$. In each panel, the co-added LCP and RCP filtergram (LCP+RCP), the magnetogram, and the Dopplergram (top to bottom) of the selected subfield is displayed. (At a heliocentric angle of $55^{\circ}$, no dark-cored filament could be clearly identified in the limb-side penumbra.) We start the tracing, where the filaments appear in the umbra - defining the inner foot points - following the structure as far as they can be identified in the outer penumbra. Figures $6-8$ show the variation in the 

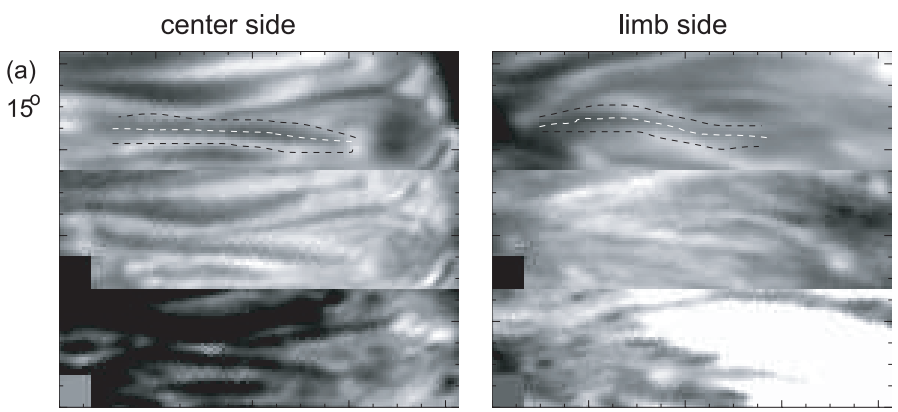

(b)

$29^{\circ}$

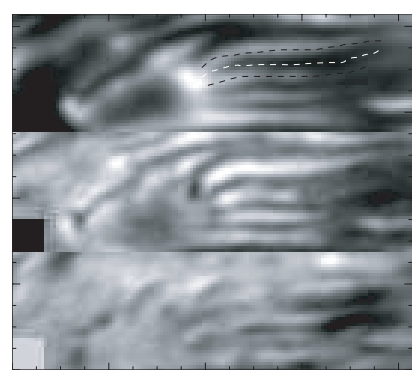

(c)

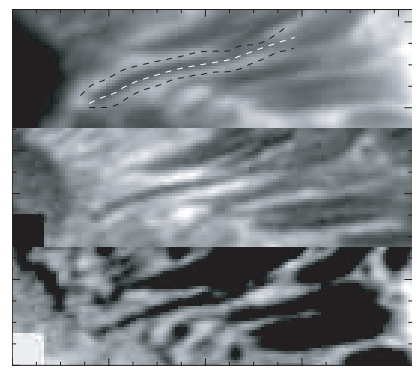

(d)

$55^{\circ}$

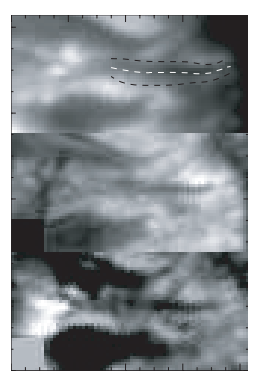

Fig. 5. Dark-cored filaments at different heliocentric positions. In each panel, $\mathrm{LCP}+\mathrm{RCP}$ is displayed in the top, the magnetogram in the middle and the Dopplergram in the bottom (left column: center-side penumbra, right column: limb-side penumbra). The squares in the lower left of the magnetograms and Dopplergrams mark the grey scale corresponding to a magnetic signal of zero and the zero point LOS velocity, respectively. a) NOAA 10655 at LOS angle of $15^{\circ}$, August 5, 2004; b) NOAA 10649 at $29^{\circ}$, July 16, 2004; c) NOAA 10653 at $42^{\circ}$ July 20, 2004; d) NOAA 10655 at 55 , August 8, 2004.

normalized intensity, the magnetogram signal, and the LOS velocity along both the dark core and the lateral brightenings indicated by dashed lines in LCP+RCP, where the dark-cored filaments are very pronounced.

\section{Intensity variation}

The intensity diagrams ${ }^{2}$ show that the intensity difference between dark core and lateral brightening is larger in the inner part of the limb-side penumbra than in the inner part of the centerside penumbra at all observed heliocentric positions. In general

2 The fact that the paths are selected in LCP+RCP explains that in some diagrams of Fig. 6 the normalized continuum intensity of the lateral brightenings is lower than the intensity of the dark core itself.
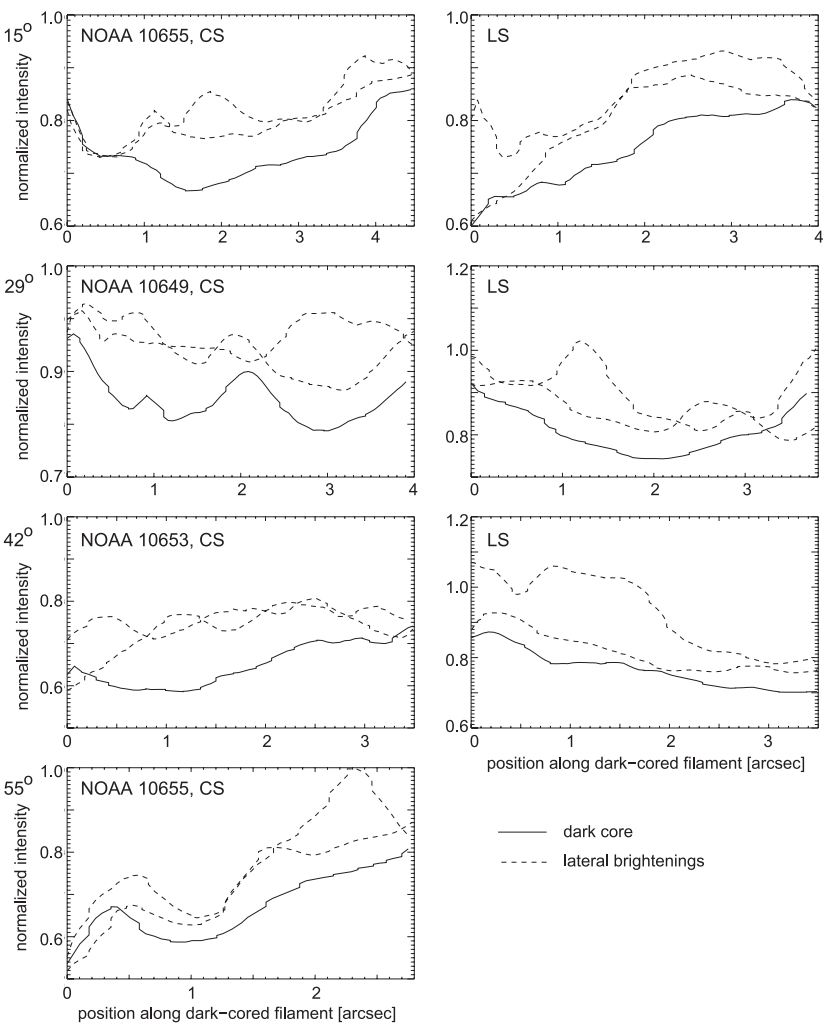

_ dark core

..... lateral brightenings

Fig. 6. Variation of the normalized intensity along selected dark-cored filaments at different heliocentric positions. CS - center side, LS limb side.

the local intensity variations (within $1^{\prime \prime}$ ) of the lateral brightenings are spatially uncorrelated with the local intensity variations in the dark cores. The general trend throughout the penumbra is similar.

\section{Magnetic field}

The lateral brightenings are always related to a stronger magnetogram signal relative to that of the dark cores throughout both the center-side and the limb-side penumbra. This is true for all observed heliocentric positions where dark cores are visible. The magnetogram signal of the center-side dark-cored filaments is stronger than for the filaments at the limb side. The absolute difference between dark-core signal and lateral brightening is smallest for the filaments observed in the limb-side penumbra at $29^{\circ}$ and $42^{\circ}$. The strongest magnetogram signal close to the foot point of a center-side dark-cored filament is observed at $55^{\circ}$. In the center-side penumbrae we do not observe a negative magnetogram signal for the dark-core component nor for the lateral brightening. In the limb-side penumbra, we measure negative magnetogram signals within the dark cores at the observed heliocentric angles of $29^{\circ}$ and $42^{\circ}$. At a heliocentric angle of $29^{\circ}$, the zero level is reached at $2^{\prime \prime} .5$ and at a heliocentric angle of $42^{\circ}$, the signal crosses zero at $2^{\prime \prime}$. At these points, the inclination of the field is $56^{\circ}$ and $48^{\circ}$ respectively. For smaller distances, the signal is positive, indicating that the magnetic field inclination increases with distance from the foot point.

\section{Velocity field}

In the center-side penumbra, the dark cores are related to a strong negative (blue shifted) LOS velocity component. For all observed heliocentric angles, the dark cores are always related 

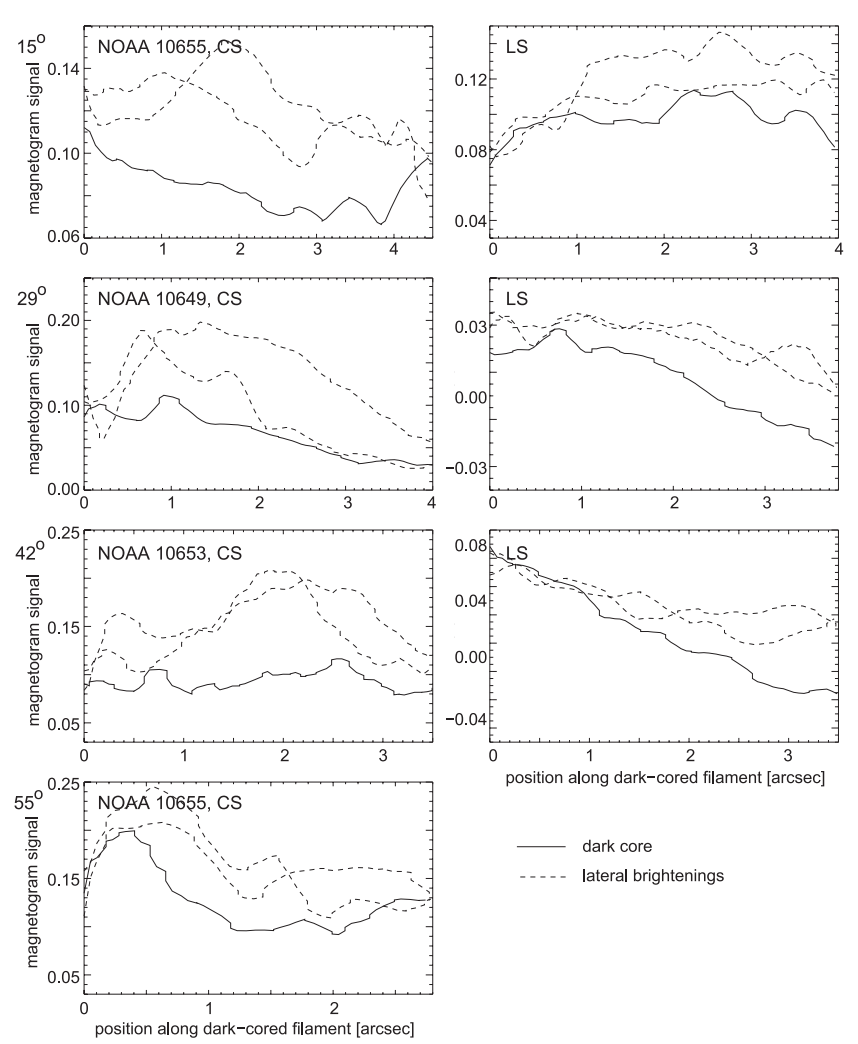

Fig. 7. Magnetogram signal variation along selected dark-cored filaments at different heliocentric position. CS - center side, LS - limb side.

to a larger absolute LOS velocity than the lateral brightenings. In general, the absolute LOS velocity increases with increasing distance from the umbra in both in the center-side and limb-side penumbra for all observed heliocentric angles. The increase is stronger for the dark cores relative to their lateral brightenings both in the center- and limb-side penumbra. This indicates that the horizontal velocity component is stronger in the dark cores than in the lateral brightenings.

For the selected dark cores, we neither observe positive LOS velocities in the center-side penumbra nor negative LOS velocities in the limb-side penumbra. The measured velocity varies strongly from one dark core to another. For example, the dark core in Fig. 5c above the selected dark core shows a stronger LOS velocity along the whole structure than the selected one. In comparison to the selected dark core these two structures are related to a much stronger velocity signal in the outer penumbra. The Dopplergram of Fig. 5d shows two elongated structures (one above and one below the selected dark-cored filaments) with strong LOS velocity but the related structure in the continuum intensity could not clearly be identified as a dark core. Additionally, the measured velocity can vary significantly along the dark core. E.g., the center-side dark core observed at $42^{\circ}$ shows a strong localized increase of the velocity for for positions along the dark-cored filaments in the range 2-3" (cf. Fig. 8).

In the limb-side penumbra, the LOS velocity of dark cores is positive, already at the location of their foot points. The inclination of the flow field is consequently larger than $(90-42)^{\circ}$ at this point. In general the measured absolute LOS velocities are lower than in the center-side penumbra. At $42^{\circ}$, the absolute LOS velocity measured at the center-side footpoint is stronger than the measured velocities at the foot points at $29^{\circ}$ and $55^{\circ}$ for both dark core and lateral brightenings. Additionally, we measure a
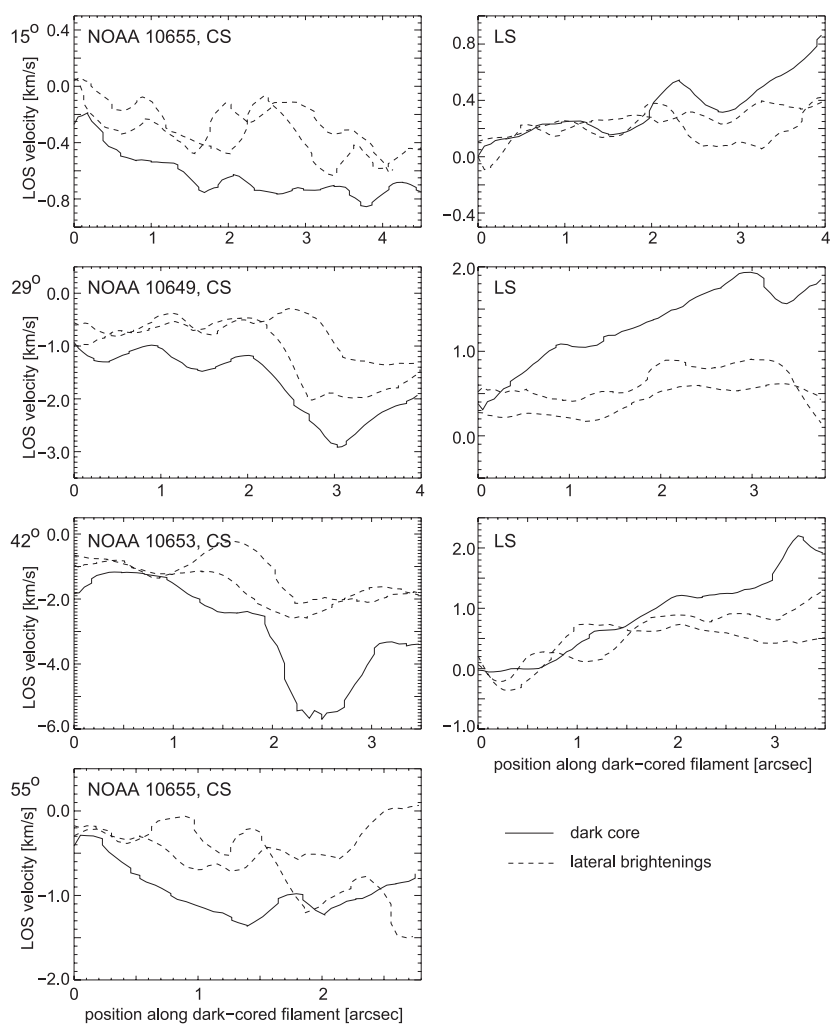

_ dark core

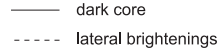

Fig. 8. LOS-velocity ( $557.6 \mathrm{~nm})$ variation along selected dark-cored filaments at different heliocentric positions. CS - center side, LS - limb side.

LOS velocity close to zero at the limb-side footpoint at $29^{\circ}$. This indicates an inclination of the velocity field between $29^{\circ}$ and $55^{\circ}$ at the dark core foot points.

At locations where dark-cored filaments split or reach far into the umbra, we observe positive (red shifted) LOS velocities in the center-side penumbra. E.g., in the upper right corner of Fig. 5a is a filament visible that splits in the umbra. The splitting is visible both in the continuum filtergram and in the magnetogram, but could not be identified in the Dopplergram that shows a negative signal at this location. Also the dark-cored filament that reaches longest into the umbra in Fig. 5b shows a discontinuous velocity signal. In the penumbra the velocity signal of this dark core is close to zero. Only in the outer penumbra, a negative LOS velocity is detected.

\subsection{Magnetic field strength and inclination in dark-cored filaments}

Accurate measurements of magnetic field vectors require full Stokes measurements at several wavelengths and modeling combined with inversion techniques. However, reasonable estimates of field strength and inclination variations across penumbral fine structure can be obtained from magnetograms. In Langhans et al. (2005), L. Bellot Rubio kindly provided radiative transfer calculations using the temperature and electron pressure stratifications of the mean penumbral model of del Toro Iniesta et al. (1994). Plotting the predicted magnetogram signal versus the temperature difference $\Delta T$ relative to the mean penumbral temperature stratification, the calculated magnetogram signal is higher at lower temperatures, $-1500 \mathrm{~K}<\Delta T<0 \mathrm{~K}$, and lower at higher temperatures, $0 \mathrm{~K}<\Delta T<1000 \mathrm{~K}$ (cf. Fig. 8 in Paper I). From this plot, it can be concluded that the difference in field strength 

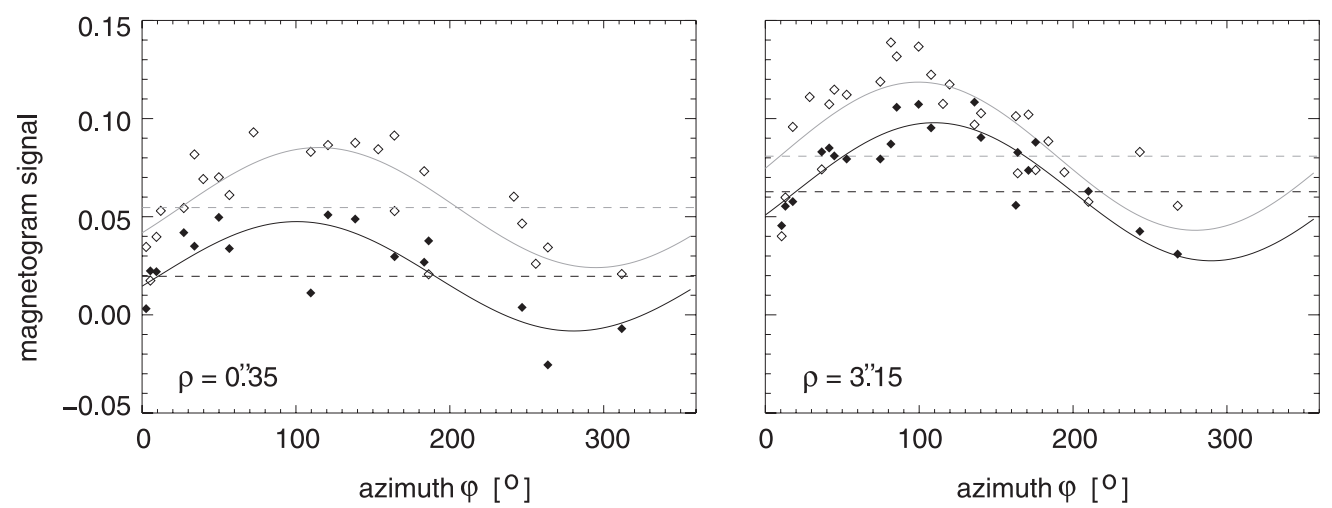

Fig. 9. Magnetic signal obtained from azimuthal slices at $\rho=0{ }^{\prime} 35$ and $\rho=3$ '” 15 , data from August $9, \mu=0.88$. Dashed lines represent $M_{\mathrm{v}}^{\vartheta}(\rho)$. Dark core data - solid diamonds, black curve. Bright edge data - open diamonds, gray curve.
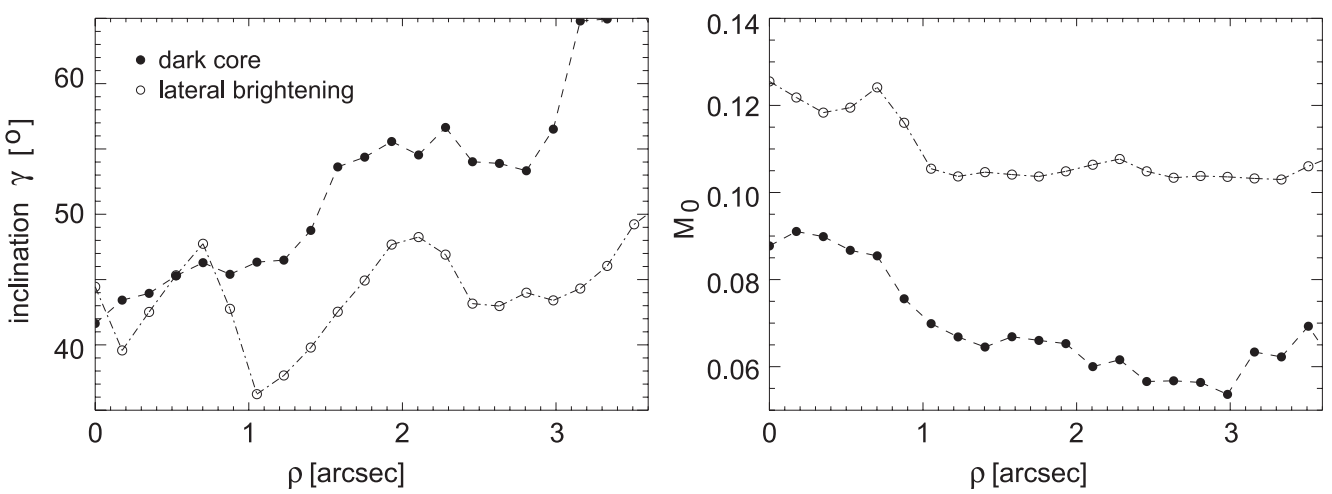

Fig. 10. Radial dependence of the magnetic field along the dark-cored filament. Left: magnetic field inclination $\gamma ;$ right: $M_{0}$.

between dark cores and their lateral brightenings may even be larger than indicated by magnetograms.

To derive estimates of the magnetic field strength and inclination we use the geometrical method described in detail in Paper I. The magnetogram signal, as defined in Eq. (1), can be written as

$M^{\vartheta}=\alpha\left(B, T, v_{\mathrm{LOS}}\right) B^{\vartheta}$.

The magnetogram signal is proportional to the longitudinal magnetic field $B^{\vartheta}$, but it is also a function of $B$ itself, the temperature $T$, and the LOS velocity $v_{\mathrm{LOS}}$. The projection of the horizontal component of $\alpha \boldsymbol{B}$ to the LOS, $M_{\mathrm{h}}^{\vartheta}(r)$, describes an azimuthal sinusoidal variation for constant $r$ with the amplitude $M_{\mathrm{h}, 0}^{\vartheta}(r)$ :

$M_{\mathrm{h}}^{\vartheta}(r)=M_{\mathrm{h}, 0}^{\vartheta}(r) \sin \varphi$.

The projection of the vertical component of $\alpha \boldsymbol{B}$ to the LOS $M_{\mathrm{v}}^{\vartheta}(r)$ does not vary with the azimuth and is represented by the offset of the sinusoidal least squares fit to $M^{\vartheta}(r, \varphi)$ for constant $r$. The inclination angle $\gamma$ is finally computed from the ratio of $M_{\mathrm{v}}^{\vartheta}(r)$ and $M_{\mathrm{h}, 0}^{\vartheta}$.

Langhans et al. (2005) use the azimuthal fluctuation in both the intensity filtergrams and the magnetograms to separate the penumbra spatially in two components and define binary masks. In this way, properties of each component can be analyzed separately. Here the dark core structures are selected by eye. The analysis is based on 26 dark-cored filaments (NOAA 10425 on August 9, 2003, UT 11.45). Because the penumbral filaments originate at different distances from spot center (over a range of at least a few arc seconds), we assign an individual radial coordinate, $\rho$, to each of the dark-cored filaments, where they originate in the umbra. We are assuming that their structure is independent of the distance from spot center. Figure 9 shows azimuthal slices taken at different values of $\rho$ for the dark cores (solid diamonds) and the bright edges (open diamonds). For the dark cores, $M_{\mathrm{v}}^{\vartheta}(\rho)$ decreases with increasing $\rho$. The amplitude of the fitted sinusoidal, $M_{\mathrm{h}, 0}^{\vartheta}(\rho)$, is of the same magnitude for both values of $\rho$. For the bright component of the dark-cored filaments $M_{\mathrm{h}, 0}^{\vartheta}(\rho)$ is also almost constant for different values of $\rho . M_{\mathrm{v}}^{\vartheta}(\rho)$ decreases less with increasing $\rho$ in comparison to the dark core magnetic field. The variation of the inclination angle $\gamma$ along $\rho$ is plotted in Fig. 10. At the foot point $\gamma$ is the same for both the dark core and the lateral brightening. While $\gamma$ increases continuously with increasing $\rho$ for the dark core, the inclination of the surroundings remain around $45^{\circ} . M_{0} \equiv \alpha B_{0}$ (right panel in Fig. 10) decreases with distance from spot center. The decrease for the bright component is small in comparison to the decrease of the dark core component (30\% within a distance of $\left.2^{\prime \prime}\right)$.

From Fig. 10 we conclude that the magnetic field strength is approximately a factor of two weaker in the dark cores compared to the lateral brightenings. As noted in Sect. 4.1, the distance between the lateral brightenings is very small, approximately $0.3-$ $0 . ' 4$. We can therefore not exclude the possibility of significant polarized stray-light and that the field strength in the dark cores is even smaller than measured. 

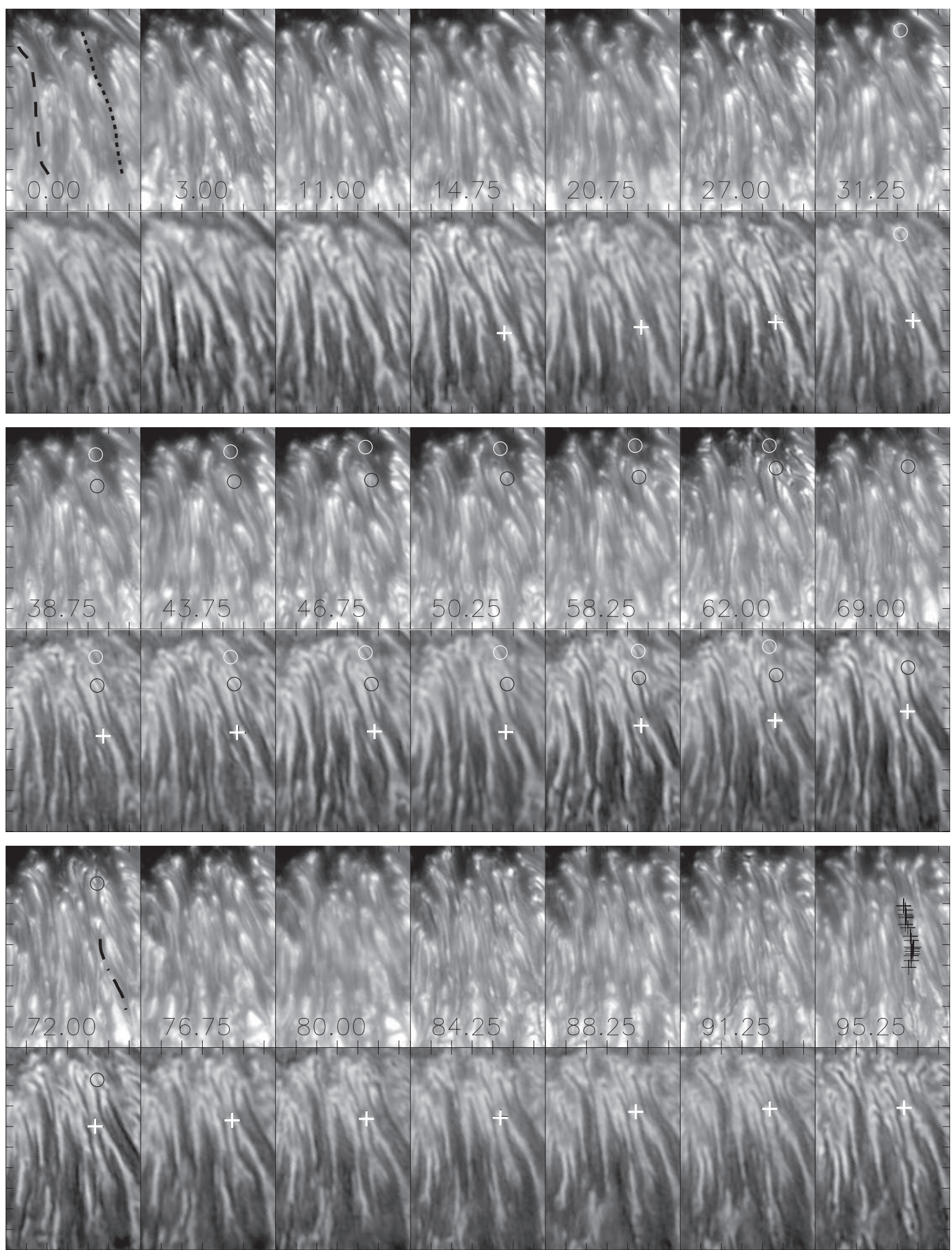

Fig. 11. Evolution of dark-cored filaments during $1 \mathrm{~h} 35 \mathrm{~min}$ in the $G$ continuum (top) and magnetogram (bottom). One tickmark corresponds to $1^{\prime \prime}$. The time stamps are given in minutes after the start of the sequence.

\subsection{Time evolution of dark-cored filaments}

The filamentary structures, wherein the dark cores are embedded, are very stable. Figure 11 shows 22 snapshots of a small part of the center-side penumbra of the symmetric sunspot of NOAA 10425 on August 8 at $\mu=0.97$, corresponding to $95 \mathrm{~min}$. We follow the evolution of three dark-cored filaments.
Filament 1 (marked with the dashed line in the first continuum frame): the selected dark core stretches over the entire penumbra during the whole observed sequence. During the sequence the inner foot point of the filamentary structure moves about 1 " sideways and in direction toward the umbra. The foot point covers a distance of about $2^{\prime \prime}$, which corresponds to an average proper motion speed of $260 \mathrm{~m} \mathrm{~s}^{-1}$, inferred from the 
displacement of the inner footpoint. In the snapshot, taken at $58.25 \mathrm{~min}$ after the beginning of the sequence (labeled 58.25), the structure starts to split in the umbra forming a Y-shape, best visible a few minutes later (62.00). In the following snapshots, the Y-shape disappears, leaving a shortened filamentary structure and a bright dot in the umbra (69.00 to 72.00). The development of a Y-shape is a quite common feature. E.g., the filamentary structure to the right of the selected filament forms a Y-shape (38.75 to 50.25), that disappears again a few minutes later. Except for this development of Y-shapes, the structure of the dark-cored filaments is very stable at least on a time scale of $1.5 \mathrm{~h}$.

Filament 2 (marked with the dotted line in the first continuum frame): in the first frames of the sequence this filament can be traced throughout the entire penumbra. From 14.75 the filament fades in the continuum. Of the lateral brightenings, only one remains visible. Whereas the dark core can no longer be identified in the continuum images, the dark-cored magnetic structure remains visible throughout the entire penumbra $(14.25$ to 27.00$)$. In the following frames (31.25 to 62.00) the magnetic signature of the filament - position marked by white circles in both magnetograms and continuum - disappears in the outer penumbra and finally fades also in the inner penumbra (69.00).

Filament 3 (marked with the dashed-dotted line in the continuum frame at 72.00): this filament is an example of a newly formed inward-moving dark-cored filament. The magnetic signature of a dark-cored filament can already be detected at 14.75, marked with a white cross. (A hint for that signature might even be identified earlier in the sequence.) In the continuum filtergrams, the dark-cored filament is not visible until 62.00. The inner foot point of this filament - marked by white crosses apparently moves inwards with an average speed of $140 \mathrm{~m} \mathrm{~s}^{-1}$.

\section{Discussion and conclusion}

We confirm the observation of Sütterlin et al. (2004) concerning the asymmetrical appearance of dark-cored filaments between limb- and center-side penumbra. The number of dark cores observed is higher in the center-side penumbra than in the limbside penumbra. However, the contrast is higher in the inner part of the limb-side penumbra, where the dark-cored filaments are frequently associated with dot-like brightenings (substructure of penumbral grains), a signature that is not visible in the center side. We find that the dark cores become hard to discern already at rather small distances off disk center. In continuum filtergrams, dark cores can be seen only at the center side penumbra for $\mu=0.57$. However, Ca II H filtergrams show dark cores even in the pass band closest to the line core formed approximately 150-200 km above the photosphere and corresponding to a continuum formation optical depth of less than 0.1 . The conclusion is that dark-cored filaments are associated with a strongly warped visible $(\tau=1)$ surface. This is consistent with our conclusion that the magnetic field strength is much weaker within the dark cores compared to the lateral brightenings, implying large horizontal gas pressure gradients. Such horizontal force balance requires that the dark core must be elevated relative to the lateral brightenings. In spite of that complex topology, the $\mathrm{Ca}$ II $\mathrm{H}$ wing images suggest that the thermal structure above the photosphere of the penumbra is simpler than above the quiet sun photosphere.

The time series of August 8, 2003 shows clearly that the dark-cored filaments are very stable. Dark-cored filaments are observed to fade gradually and to be "replaced" or "masked" by new neighboring dark-cored filaments that appear to move towards spot center from the outer penumbra. This process is very similar to that of the flow channels described by Rimmele \& Marino (2006). Frequently, dark-cored filaments develop Yshapes in the penumbral-umbral boundary, leaving a bright dot in the umbra but remaining unchanged in the rest of the penumbra. This shortening of dark-cored filaments in the inner penumbra could be interpreted as a disappearance of the filament at lower spatial resolution, possibly explaining the observed life time of less than 45 min for dark-cored filaments found by Sütterlin et al. (2004).

Dark-cored filaments are visible in all spectral bands investigated here, but the structures are most obvious and also most stable in magnetograms. Even in the limb-side penumbra at the large heliocentric angles, where dark-cored filaments can hardly be identified in filtergrams, magnetograms show the characteristic signature of dark-cored filaments.

We do not find any radial variation in the magnetogram signal of the lateral bright components of the dark-cored filaments that is correlated to the observed intensity variations. Nor do we find a counterpart in magnetograms and Dopplergrams to the asymmetry of individual dark-cored filaments observed in intensity filtergrams, i.e., that one lateral brightening is significantly brighter than the other (cf. Scharmer et al. 2002).

Our observations show that the dark cores are related to a more horizontal magnetic field than their lateral brightenings. Following our analysis in Sect. 4.4, the dark cores are also related to a strongly reduced magnetic field strength.

Borrero et al. (2006b) interpreted observed Stokes spectra within the framework of a two-component model consisting of a flux tube and a background atmosphere. Within that framework, Borrero et al. found that in the inner penumbra the flux tubes have field strengths that are up to a factor three lower than for the surrounding atmosphere and that the magnetic field in the flux tube is more horizontal by about $40^{\circ}$ than in the surroundings, but still inclined by $25^{\circ}-30^{\circ}$ with respect to the horizontal. They also found that the Evershed flow is associated with the flux-tube component and is virtually absent in the surrounding atmosphere. These properties are in good overall agreement with the analysis presented here if we identify the flux tube component with the dark core and the surrounding atmosphere of Borrero et al. with the lateral brightenings of our observed filaments. The inclination of $25^{\circ}-30^{\circ}$ with respect to the horizontal implies that a flux tube would ascend by approximately $100 \mathrm{~km}$ along a radial distance of only $200 \mathrm{~km}$ and would be identifiable as an individual flux tube over a small radial distance only.

Direct measurements of the magnetic field strength - based on spectra of the Fe II $614.9 \mathrm{~nm}$ line - by Bellot Rubio et al. (2005) show that dark-cored penumbral filaments do exhibit weaker fields than their surroundings (but only by 100-300 G) and that the dark cores show weaker fields than their lateral brightenings.

The inclination of the dark-core magnetic field increases from $40^{\circ}$ to $60^{\circ}$ within a few arc seconds distance from their foot points in the umbra. The bright component shows very little radial variation both in inclination and in the strength of the magnetic field. In the analysis in Paper I, the increase takes place within a shorter distance, probably because the azimuthal average over different radial parts of individual dark cores was calculated at a fixed radial distance.

The dark cores are related to larger absolute LOS velocities than their immediate surroundings. From observations at different heliocentric position angles, we conclude that the velocity field has a strong horizontal component within the dark cores. This is in accordance with recent high-resolution observations 
of Rimmele \& Marino (2006). The Evershed flow is obviously concentrated in the dark cores. The velocity signal within the dark cores varies considerably from one dark-cored filament to another. In addition, we frequently observe radially localized enhancements of the LOS velocity within individual dark cores, suggesting that the gas outflow may take place in "packages" (cf. Shine et al. 1994; Rimmele 1994; Rouppe van der Voort 2003). Rimmele \& Marino (2006) explain radially localized enhancements of the LOS velocity by "stacked velocity channels", however none of our highly resolved images support the existence of crossed filaments.

An important clue to understanding the dynamics and magnetic fields of penumbra is provided by their connection to peripheral umbral dots (Scharmer et al. 2002). These are shown by Rimmele \& Marino (2006) to constitute the inner foot points of flow channels and interpreted by them as support for the moving tube model of Schlichenmaier et al. (1998a,b). The splitting of dark cores into Y-shapes described here is a particularly interesting process, arguing against the identification of dark-cored filaments with flux tubes. Schüssler \& Vögler (2006) have made full 3D MHD simulations, including gray radiative transfer, for an initially uniform vertical field with a field strength of $2500 \mathrm{G}$. Within that atmosphere, convection develops in the form of narrow upflow plumes with adjacent downflows that become almost field-free near the surface. Interestingly, the so-formed umbral dots in most cases develop an elongated shape with a central dark lane. In many cases, larger dots show a threefold dark lane, quite similar to the Y-shaped dark cores found in our observed filaments protruding into the umbra. The dark lanes in the simulations of Schüssler \& Vögler are due to the same opacity effect as proposed by Spruit \& Scharmer (2006) to explain the penumbra dark cores: that the formation height is shifted into the higher and cooler layers of the atmosphere by the (nearly) field-free gas. This model is consistent with the analysis presented here, showing weaker and more horizontal magnetic fields at locations of the dark cores as compared to the surroundings. Within the concept of this model, the evolution of dark cored filaments described by our observations and by Rimmele \& Marino (2006) can be interpreted as a continuous opening and closing of fieldfree gaps. Our objections to the interpretation of Rimmele \& Marino are partly that they do not demonstrate that their observed flow channels are horizontal and partly based on theoretical arguments: It is very difficult to understand how flux tubes with diameters in the range $0.2-0{ }^{\prime} 4(150-300 \mathrm{~km})$ can provide the energy needed to explain the radiative output of filaments that are nearly $4000 \mathrm{~km}$ long, in particular since the measured upflow velocities are only on the order of $1 \mathrm{~km} \mathrm{~s}^{-1}$.

The data presented are consistent with the interpretation of the dark cores as signatures of convection in field-free radially aligned gaps just below the visible surface (Spruit \& Scharmer 2006). This allows a natural explanation to the classical heat flux problem of penumbrae, whereas the flux tube interpretation in this context leads to severe problems. There is also supporting evidence from MHD simulations (Nordlund, private communication) that explain dark lanes in light bridges (Lites et al. 2004) as the result of field-free gas protruding into magnetic gas and producing a dark lane by the combined effect of a locally elevated $\tau=1$ surface and an overall drop of temperature with height. Additional evidence for the gappy penumbra model can be found in the work by Löfdahl et al. (2006), demonstrating a direct connection between dark lanes in light bridges and darkcored penumbral filaments.
Acknowledgements. The Swedish 1-m Solar Telescope is operated on the island of La Palma by the Royal Swedish Academy of Sciences in the Spanish Observatorio del Roque de los Muchachos of the Instituto de Astrofísica de Canarias. K. Langhans' research is funded by the European Community's Human Potential Program through the European Solar Magnetism Network (contract HPRN-CT-2002-00313).

\section{References}

Balthasar, H. 1985, Sol. Phys., 99, 31

Bellot Rubio, L. R., Balthasar, H., \& Collados, M. 2004, A\&A, 427, 319

Bellot Rubio, L. R., Balthasar, H., Collados, M., \& Schlichenmaier, R. 2003, A\&A, 403, L47

Bellot Rubio, L. R., Langhans, K., \& Schlichenmaier, R. 2005, A\&A, 443, L7 Berger, T. E., \& Title, A. M. 2001, ApJ, 553, 449

Borrero, J. M. 2004, Ph.D. Thesis, University of Göttingen

Borrero, J. M., Lagg, A., Solanki, S. K., \& Collados, M. 2005, A\&A, 436, 333

Borrero, J. M., Rempel, M., \& Solanki, S. K. 2006a, [ArXiv: astro-ph/0602129]

Borrero, J. M., Solanki, S. K., Bellot Rubio, L. R., Lagg, A., \& Mathew, S. K. 2004, A\&A, 422, 1093

Borrero, J. M., Solanki, S. K., Lagg, A., Socas-Navarro, H., \& Lites, B. 2006b, A\&A, 450, 383

Carlsson, M., Stein, R. F., Nordlund, Å., \& Scharmer, G. B. 2004, ApJ, 610, L137

del Toro Iniesta, J. C., Tarbell, T. D., \& Ruiz Cobo, B. 1994, ApJ, 436, 400

Jahn, K., \& Schmidt, H. U. 1994, A\&A, 290, 295

Langhans, K. 2006, in Proc. 4th Solar Polarization Workshop, ASP Conf. Ser., in press

Langhans, K., Scharmer, G. B., Kiselman, D., Löfdahl, M. G., \& Berger, T. E. 2005, A\&A, 436, 1087

Lites, B. W., Scharmer, G. B., Berger, T. E., \& Title, A. M. 2004, Sol. Phys., 221,65

Löfdahl, M. G. 2002, in Proc. SPIE, Vol. 4792, Image Reconstruction from Incomplete Data II, ed. P. J. Bones, M. A. Fiddy, \& R. P. Millane, 146

Löfdahl, M. G., Langhans, K., Narayan, G., \& Scharmer, G. B. 2006, A\&A, in preparation

Martínez Pillet, V. 2000, A\&A, 361, 734

Meyer, F., \& Schmidt, H. U. 1968, Mitteilungen der Astronomischen Gesellschaft Hamburg, 25, 194

Müller, D. A. N., Schlichenmaier, R., Steiner, O., \& Stix, M. 2002, A\&A, 393, 305

Rimmele, T., \& Marino, J. 2006, ApJ, 646, 593

Rimmele, T. R. 1994, A\&A, 290, 972

Rouppe van der Voort, L. H. M. 2002, A\&A, 389, 1020

Rouppe van der Voort, L. H. M. 2003, A\&A, 397, 757

Rouppe van der Voort, L. H. M., Löfdahl, M. G., Kiselman, D., \& Scharmer, G. B. 2004, A\&A, 414, 717

Sütterlin, P., Bellot Rubio, L. R., \& Schlichenmaier, R. 2004, A\&A, 424, 1049

Scharmer, G. B., Bjelksjö, K., Korhonen, T. K., Lindberg, B., \& Pettersson, B. 2003a, in Innovative Telescopes and Instrumentation for Solar Astrophysics, ed. S. Keil, \& S. Avakyan, Proc. SPIE, 4853, 341

Scharmer, G. B., Dettori, P., Löfdahl, M. G., \& Shand, M. 2003b, in Innovative Telescopes and Instrumentation for Solar Astrophysics, ed. S. Keil, \& S. Avakyan, Proc. SPIE, 4853, 370

Scharmer, G. B., Gudiksen, B. V., Kiselman, D., Löfdahl, M. G., \& Rouppe van der Voort, L. H. M. 2002, Nature, 420, 151

Schlichenmaier, R. 2002, Astron. Nachr., 323, 303

Schlichenmaier, R., Jahn, K., \& Schmidt, H. U. 1998a, ApJ, 493, L121

Schlichenmaier, R., Jahn, K., \& Schmidt, H. U. 1998b, A\&A, 337, 897

Schlichenmaier, R., \& Schmidt, W. 2000, A\&A, 358, 1122

Schlichenmaier, R., \& Solanki, S. K. 2003, A\&A, 411, 257

Schmidt, W., \& Fritz, G. 2004, A\&A, 421, 735

Schüssler, M., \& Vögler, A. 2006, ApJ, 641, L73

Shine, R. A., Title, A. M., Tarbell, T. D., et al. 1994, ApJ, 430, 413

Solanki, S. K., \& Montavon, C. A. P. 1993, A\&A, 275, 283

Spruit, H. C., \& Scharmer, G. B. 2006, A\&A, 447, 343

Thomas, J. H. 1988, ApJ, 333, 407

Title, A. M., Tarbell, T. D., Topka, K. P., et al. 1989, ApJ, 336, 475

Vernazza, J. E., Avrett, E. H., \& Loeser, R. 1981, ApJS, 45, 635

Wentzel, D. G. 1992, ApJ, 388, 211 